\title{
Mental Health Over Time and Financial Concerns Predict Change in Online Gambling During COVID-19
}

\author{
Alex Price ${ }^{1,2}$ (D) Nassim Tabri ${ }^{2,3} \cdot$ Sasha Stark $^{1,2} \cdot$ Iris M. Balodis $^{2,4,5}$. \\ Michael J. A. Wohl' ${ }^{2,3}$
}

Accepted: 27 December 2021

(c) The Author(s), under exclusive licence to Springer Science+Business Media, LLC, part of Springer Nature 2022

\begin{abstract}
This study examined online gambling behavior during COVID-19 land-based gambling restrictions and associations with changes in mental health, impacts on household income due to the pandemic, financially focused motivations, and symptoms of gambling problems. A repeated online survey was administered to adult gamblers in Ontario, Canada. Wave 1 was conducted at the beginning of the first COVID-19 lockdown (April 2020) and wave 2 in August 2020. Respondents $(N=940)$ completed validated self-report questionnaires related to depression and anxiety, financial motivation, financial impacts due to the pandemic, online gambling behaviors, and problem gambling symptomatology. A cluster analysis identified three subgroups: no online gambling (subgroup 1), engagement in a range of gambling games online and change in gambling involvement (i.e., some increased and some decreased gambling; subgroup 2), and predominantly online lottery play with no change in online gambling (subgroup 3). Respondents who reported increased anxiety and depression symptom severity between the two waves and those who experienced greater symptoms of problem gambling and negative impacts on household income due to COVID-19 were most likely to be found in subgroup 2. Greater financial focus was also noted in this group. Results indicate a link between change in online gambling involvement during COVID-19 and increased mental health problems, elevated problem gambling severity, negative impacts on household income, and a greater financially focused self-concept. These results may help generate novel research questions examining short and longterm effects of the pandemic on online behaviors that inform policy and practice.
\end{abstract}

Keywords Online gambling · COVID-19 $\cdot$ Mental health $\cdot$ Financial impact · Financial motivation

In December 2019, a novel coronavirus disease (COVID-19) emerged in Wuhan, China, leading to a global pandemic. As of November 2021, over 255 million cases of COVID19 have been confirmed worldwide with more than 5 million people having died from the virus (World Health Organization, 2021). Lockdown measures enacted to control the virus

Alex Price

alexp@rgco.org

Extended author information available on the last page of the article 
spread (e.g., physical distancing, business shuttering, school and recreational closures) have contributed to negative secondary social, economic, and mental health consequences (Nicola et al., 2020). For instance, the pandemic has contributed to a reported increase in addictive behavior in the general population, including an increase in online gambling (Gambling Commission, 2020; Mallet et al., 2021; Neill et al., 2020; Sun et al., 2020). What is not certain or fully understood to date are the extended and general impacts of COVID-19 on gambling problems and associated comorbidities (Brodeur et al., 2021; Hodgins \& Stevens, 2021).

Although gambling is merely a form of entertainment for most of the nearly 1.6 billion people who gamble, it is estimated that millions of those people experience significant economic, social, and health harm as a result of their gambling (Calado \& Griffiths, 2016). The COVID-19 pandemic may have been particularly harmful for gamblers at high risk for gambling problems because they are more likely to be experiencing comorbid mental health problems and financial stress (Håkansson, 2020; Price, 2020). Additionally, highrisk gamblers tend to be financially motivated (i.e., they gamble to make money; Dechant, 2014; for recent review and meta-analysis, see Tabri, Philander, et al., 2021; Tabri, Xuereb, et al., 2021) despite the fact that the odds of success are not in the gambler's favor. The impacts of COVID-19 may be particularly harmful to those who are financially motivated because perceived economic disadvantage-which has increased during the COVID-19 pandemic - interacts with the desire to gamble for financial reasons and facilitates problematic gambling behavior (Tabri et al., 2017a; Tubadji et al., 2021; Hodgins \& Stevens, 2021). As such, financial motivations hold the potential to inform our understanding of gambling behaviors and outcomes during COVID-19.

Importantly, the harms that stem from gambling are not restricted to those who gamble problematically. Lower-risk gamblers also experience gambling-related harms and, perhaps counterintuitively, experience a greater proportion of the harms that stem from gambling (Browne \& Rockloff, 2018). Moreover, gambling risks and harms can radiate outwards, affecting family members, friends, employers, and communities (Alexander Blaszczynski, 2009; Browne et al., 2016; Langham et al., 2016). There is some indication that gamblingrelated harms experienced by all these parties have been exacerbated as a result of the COVID-19 pandemic due, in part, to the isolation, confinement, fear of disease, and the availability of online gambling (Hakansson et al., 2020).

In the current research, we used a repeated-measures design (two waves separated by three months) to better understand gambling and its correlates during COVID-19 precipitated closure of land-based casinos and community gambling facilities, which occurred six weeks prior to the first wave of data collection. Specifically, we assessed online gambling behaviors, problem gambling severity, mental health problems, and financial concerns and motivations among gamblers during COVID-19 corresponding with the closure of most land-based gambling venues in the province of Ontario, Canada. Collected data represented two time points over the course of three months. During the first wave of survey research, Ontario had just entered its first province-wide lockdown, which severely restricted movement and access to public and private venues, including land-based gambling establishments (e.g., casinos and community gaming facilities). The second wave of research occurred during a period of restriction easing, although most land-based gambling venues, such as casinos, remained closed.

In this study, our stated hypotheses tested self-reported change in online gambling. Change refers not only to increased or decreased frequency of play, but also games played, and change in environment (e.g., online). Specifically, we predicted that participants who reported an increase (relative to decrease) in mental health problems during the first few 
months of the COVID-19 lockdown would report greater change in online gambling during the COVID-19 land-based gambling restrictions at wave 2. In addition, we predicted that people who reported their household income as being negatively affected by COVID19 would report greater change in online gambling during the pandemic gambling restrictions compared to people who reported negligible impacts on household income. We also hypothesized that people who gambled and who are more (relative to less) financially focused - the belief that the possession of money is a central domain to self-definition and thus self-worth-would report greater changes in online gambling involvement during COVID-19 gambling restrictions. Lastly, we tested the supposition that those who have more (relative to less) symptoms of gambling problems would report greater change in online gambling during the COVID-19 gambling restrictions.

\section{Transparency Statement}

Hypotheses and the data analytic plan were pre-registered via the Open Science Framework (OSF): https://osf.io/wcqbf. For the sake of transparency, data was collected prior to pre-registration but not analyzed until after pre-registration occurred. All materials and data are available via OSF. Participants also completed other measures related COVID19 experiences. The present study focuses only on those variables relevant to the abovedescribed hypotheses.

\section{Methods}

In this study, we assessed a cohort of adult gamblers in Ontario $(n=940)$ who initially responded to an online survey (wave $1=$ April 21 to 28, 2020) during the first instance of strict lockdown measures - a time of substantial economic disruption and restrictions on recreational activity, including land-based gambling. This cohort then provided follow-up responses to an online survey administered after the easing of some lockdown measures, while over $90 \%$ of casinos remained closed (wave $2=$ August 1 to 19, 2020).

\section{Participants and Procedure}

Follow-up recruitment of adult gamblers (18 years and older) in Ontario $(n=1,018)$ was conducted by Delvinia, an online survey sample provider located in Toronto, Ontario, Canada, that served as the sole panel provider for the entire study. Utilizing their AskingCanadians panel population of over one million Canadians, Delvinia sent out recruitment notifications to registered members who had responded to the first wave survey, conducted at the end of April, 2020. Respondents recruited were directed to the research team's Qualtrics survey, where data was collected and verified for quality assurance. Return respondents were again screened before filling out the survey to ensure they were at least 18 years old, residents of Ontario, and had gambled at least once in the past 12 months, either on land or online. Survey respondents also received an informed consent statement prior to the survey and a debriefing statement at the end. This research received ethics approval from Carleton University Research Ethics Board-B (project ID\#113017). Following questionnaire 
completion, Delvinia compensated participants through a variety of consumer points programs (e.g., Hudson Bay Company, Aeroplan, VIA Preference, Petro Points, etc.). ${ }^{1}$

Of the 1,018 participants who completed both waves, 73 were excluded due to missing responses on key measures examined in the current research, three were excluded because they indicated they "did not know" when responding to a question about whether their income was affected by COVID-19, and two participants were excluded because they provided conflicting responses on the measure of online gambling (e.g., spent more money on gambling and spent less money on gambling). Thus, 940 participants were included in the analyses.

\section{Measures}

\section{Mental Health Problems}

Mental health problems were assessed through the severity of depression and generalized anxiety symptoms. Depression was assessed through the Patient Health Questionnaire (PHQ-9), derived from the Primary Care Evaluation of Mental Disorders (PRIME-ED) diagnostic tool for mental disorders (Homans, 2012; Kroenke et al., 2001). The PHQ-9 includes nine items from the Diagnostic and Statistical Manual of Mental Disorders, fourth edition (DSM-IV) depression criteria, which are scored out of a total possible 27 points. Moreover, the PHQ-9 shares a prompting question with the tool assessing anxiety symptoms, the General Anxiety Disorder (GAD-7) questionnaire: "Over the last 2 weeks, how often have you been bothered by any of the following problems?" Interpretation of depression scores include minimal (0-4), mild (5-9), moderate (10-14), moderately severe (15-19), and severe (20-27). The GAD-7 presents seven DSM-IV items for generalized anxiety disorder (Spitzer et al., 2006). Scoring was out of a total possible 21 points and features four interpretive categories, including minimal (0-4), mild (5-9), moderate (10-14), and severe (15-21). Change in mental health problems was calculated by averaging depression and anxiety scores at waves $1\left(\alpha_{\text {depression }}=0.89\right.$ and $\left.\alpha_{\text {anxiety }}=0.92\right)$ and 2 $\left(\alpha_{\text {depression }}=0.92\right.$ and $\left.\alpha_{\text {anxiety }}=0.93\right)$, and subtracting the average score of wave 2 from wave 1. As such, higher scores reflect worsening mental health.

\section{Financial Motivation}

Financial motivation was viewed as an extension of a financially focused self-conceptthe belief that self-definition and self-worth are primarily tied to financial success-and assessed via the validated Financially Focused Scale (Tabri, Will Shead, et al., 2017; Tabri, Wohl, et al., 2017; Tabri et al., 2017a). The short 4-item version was administered in wave 2 and assessed responses to key statements, including "How I feel about myself is largely based on the amount of money I have," "My moods are influenced by the amount of money I have," "People will think less of me if I don't have a lot of money," and "The opportunities that are available to me depend on the amount of money I have." The factor structure of the 4-item version was recently replicated using confirmatory factor analysis

\footnotetext{
${ }^{1}$ Points are not redeemable for cash and cannot be applied to gambling activities and the level of compensation is based on the length of the survey (in time spent on average); the specific details of which are not shared publicly.
} 
and was shown to have strict temporal measurement invariance among community gamblers over a one-month period (Tabri et al., 2021a, 2021b). Response options for each item were anchored at "not at all" (1) and "extremely" (5). The items were averaged with higher scores indicating greater financial focus $(\alpha=0.84)$.

\section{Financial Impacts}

Self-reported financial impacts during the COVID-19 pandemic were assessed in wave 2 via a 7-point Likert scale pertaining to household income. Respondents indicated to what degree they agreed or disagreed with the statement: "My household income has been negatively affected by COVID-19." Participants could also indicate "don't know" as a response; these individuals were excluded from the analysis.

\section{Online Gambling Involvement}

In wave 2, participants indicated whether they had engaged in 13 different gambling activities (see Table 1). Participants who reported involvement in at least one gambling activity were also asked to report whether they spent "more" or "less" money and "more" or "less" time gambling since wave 1 (i.e., the beginning of the COVID-19 lockdown). They were also asked if they played a "larger number," a "smaller number," or online gambling games they "never played before" the "COVID-19 gambling venue restrictions."

\section{Gambling Problems}

The Problem Gambling Severity Index (PGSI; Ferris \& Wynne, 2001) assessed gambling problems over the past 12 months through nine items scored out of a total 27 points. Interpretive categories include non-problem gambler (0), low-risk gambler (1-2), moderate-risk gambler (3-7), and high-risk problem gambler (8-27). The tool has good internal reliability in the first wave of the current research $(\alpha=0.95$; Price, 2020).

\section{Results}

Descriptive statistics for demographic and psychological factors and online gambling behaviors are reported in Table 1 . Just over half of participants indicated playing the lottery online $(53.3 \%$ ) and close to a quarter indicated playing instant lottery online (23.2\%). Other online games were endorsed to a lesser degree (see Table 1). Based on the PGSI scoring criteria, most participants did not have gambling problems $(n=688 ; 73.2 \%)$, some were at low risk for gambling problems $(n=127 ; 13.5 \%)$, few were at moderate risk for gambling problems $(n=59 ; 6.3 \%)$, and few were at high risk for gambling problems $(n=66$; $7 \%$ ). PGSI, change in mental health problems, FFS, and reports of a decrease in household income due to COVID-19 were positively intercorrelated and of small-to-moderate magnitude (see Table 2).

\section{Cluster Analysis of Online Gambling Involvement During COVID-19}

All items used to assess online gambling involvement were included in a two-step cluster analysis. In two-step cluster analysis, the data are pre-clustered in step 1. That is, a 


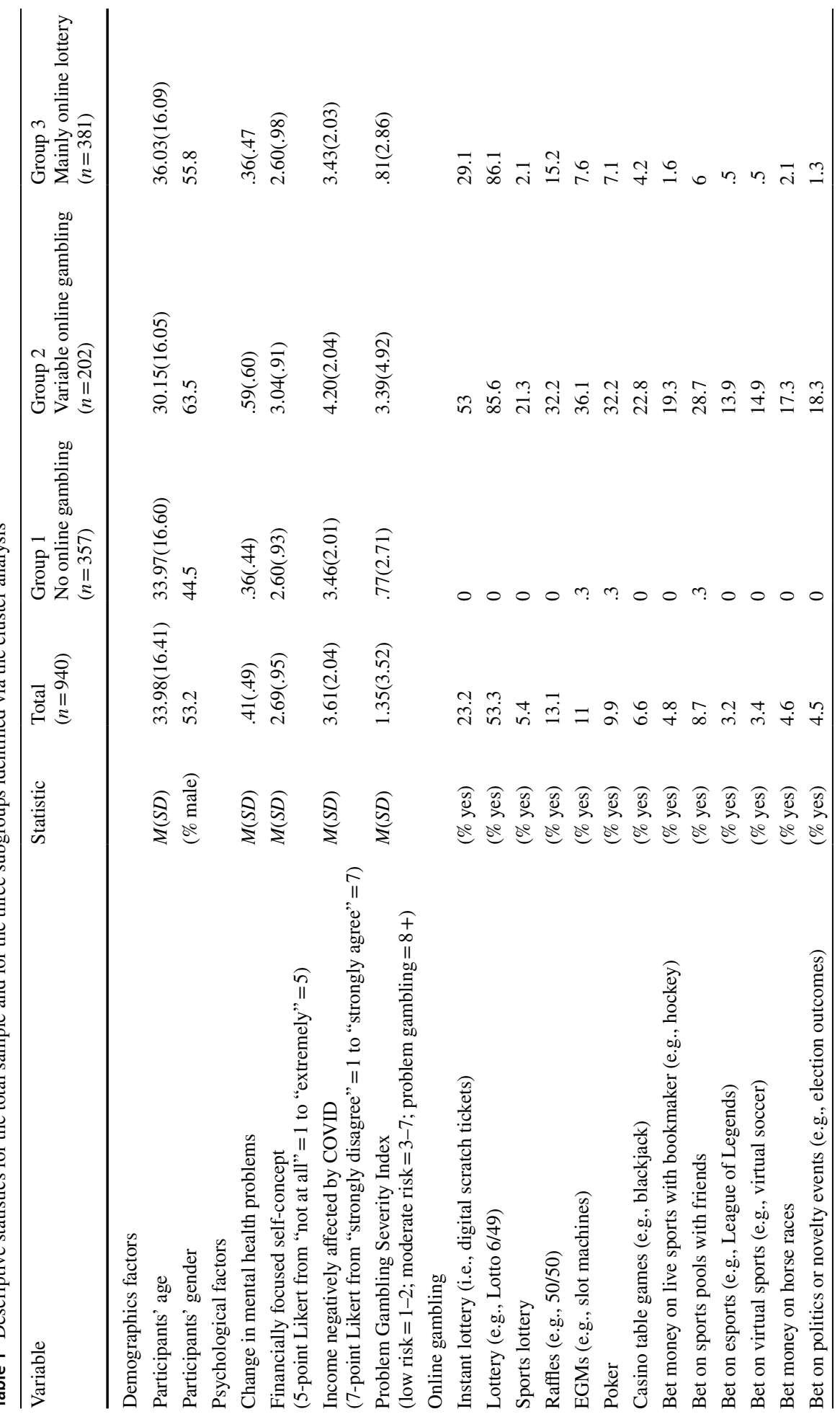




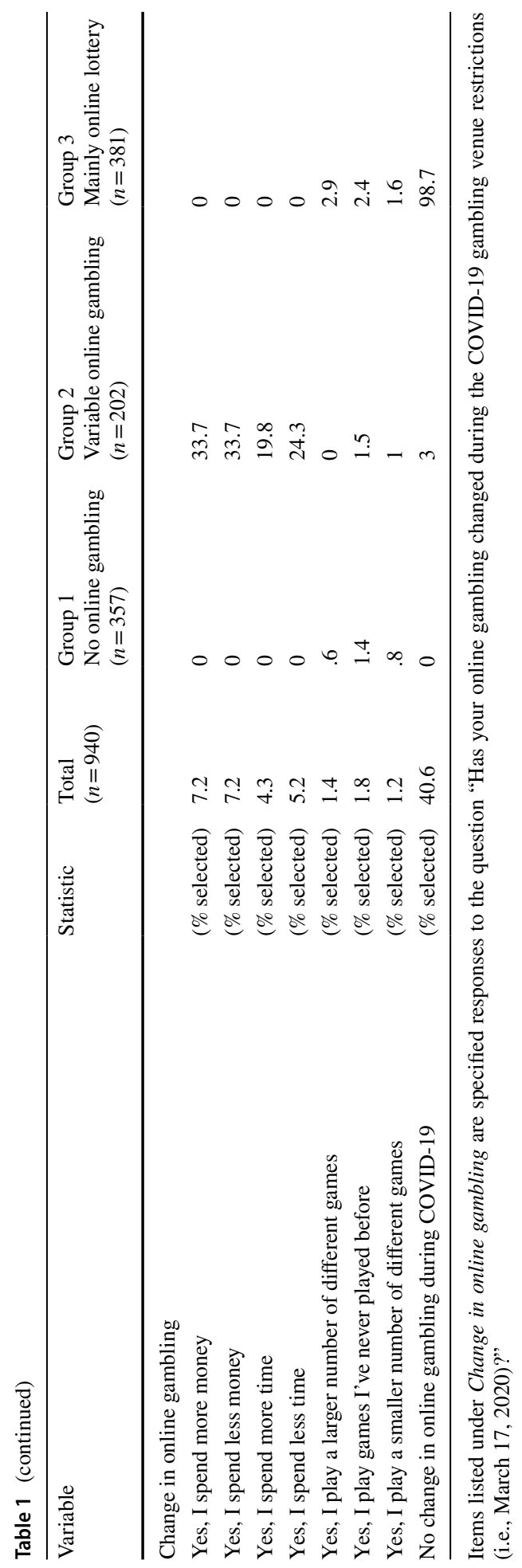


Table 2 Correlations between the predictor variables

\begin{tabular}{lllll}
\hline Variable & 1 & 2 & 3 & 4 \\
\hline 1. Change in mental health problems & - & & & \\
2. Financially focused self-concept & $.32^{* *}$ & - & & \\
3. Income negatively affected by COVID & $.23^{* *}$ & $.19^{* *}$ & - & \\
4. Problem Gambling Severity Index & $.16^{* *}$ & $.25^{* *}$ & $.15^{* *}$ & - \\
\hline${ }^{* *} p<.01$ & & & & \\
$N=940$ & & & &
\end{tabular}

data matrix is prepared in step 1 that is used in step 2. In step 2, a model-based hierarchical technique is applied to the data matrix from step 1. The log-likelihood distance was used to automatically identify clusters in the data as all variables described above, in the Methods section, were nominal categorical. The results indicated that there were three participant subgroups defined by different online gambling behaviors (see Table 1). Almost all subgroup 1 participants $(n=357)$ did not play online games. All subgroup 2 participants $(n=202)$ played a wide range of online games during COVID-19 including the lottery, EGMs, poker, and sports betting. They also indicated changes in their online play during COVID-19 (e.g., money and time spent on their activities and activities played). Subgroup 3 participants $(n=381)$ played mainly the lottery online and indicated no change in their online gambling compared to subgroup 2 participants.

\section{Hypotheses Tests}

A multinomial regression determined online gambling group membership as a function of the predictors. Univariate analyses examined the zero-order relations (one for each predictor; see Table 3). A multivariate analysis examined the unique relation between each predictor and online gambling group membership controlling for shared variance between predictors (see Table 4). In the analyses, the reference group was subgroup 3 (played online games, largely the lottery, but did not report a change in online gambling activities) and we examined the likelihood of participants being classified in subgroups 1 (did not play online games) and 2 (played online games during COVID-19 and indicated changes in online play).

Unstandardized regression coefficients and odds ratios for univariate associations are reported in Table 3 and reported for multivariate associations in Table 4. In line with our hypotheses, an increase in mental health problems from wave 1 to wave 2 , greater financially focused self-concept, reporting that income was negatively affected by COVID-19, and greater problem gambling severity were each associated with change in online gambling and greater probability of subgroup 2 membership (online multi-game players that changed their online activities) relative to subgroup 3 (online lottery players). The magnitude of the relations was small. In the multivariate analyses, the strength of the relation between each predictor and group membership was slightly attenuated but remained statistically significant.

None of the predictors differentiated between participants in subgroup 1 (who do not play online games and did not change their behavior during the COVID-19 data collection period) and participants in subgroup 3 (people who largely played the lottery online and reported no change in their online play). 


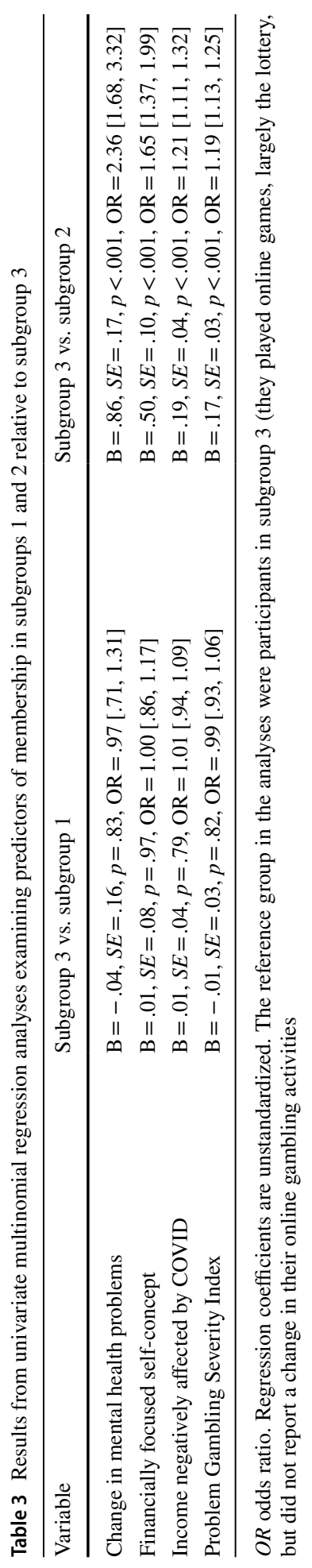




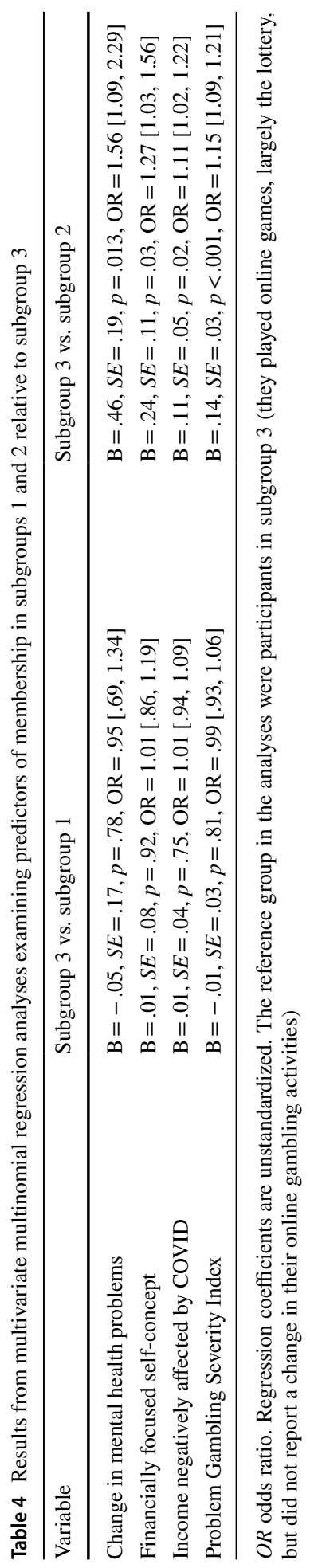




\section{Discussion}

During the entry and exit of the first lockdown in Ontario, Canada, this study found that variation in online gambling involvement during the COVID-19 pandemic was associated with increased mental health problems over time, negative household income impacts due to COVID-19, stronger financially focused self-concept, and elevated gambling problems. Three subgroups of online gamblers were identified and characterized as generally abstaining from online gambling during COVID-19 (subgroup 1); active online gamblers demonstrating greater changes of time, money, and game involvement (subgroup 2); and those who gambled online during COVID-19, but reported no change in their level of involvement and mostly played lottery (subgroup 3 ). None of the predictors was able to differentiate between subgroups 1 and 3 .

Recent studies examining the COVID-19 influences on online gamblers have reported mixed outcomes - some studies report increased gambling involvement, whereas others describe a decrease (Fluharty et al., 2020; Frisone et al., 2020; Håkansson, 2020; Price, 2020; Sharman et al., 2021). This study's findings, using a cluster analysis approach, provide some context for previous investigation's varied results. Specifically, we found a distinct at-risk cohort who report variable gambling involvement (subgroup 2), which may relate to their increased mental health problems over time, in addition to financial stress and focused motivation. Indeed, while some increase gambling to cope with negative lived experiences (e.g., poor mental health and financial troubles; Dechant, 2014; Schellenberg \& Mcgrath, 2016), others retreat from activities following long-term negative conditions like depression (Lewinsohn \& Libet, 1972). The study herein also complements one Australian study that did not find associations between problem gambling, gambling involvement, or psychological distress (Gainsbury et al., 2020) - that study reported moderate risk gamblers had a greater likelihood of increased gambling frequency compared to non-problem and low risk gamblers. The lack of associations in Gainsbury and colleagues' (2020) study may be due to the current study's use of a repeated-measurement design in which participants reported current states rather than relying on recall over multiple months. Our analytic approach also included financial impact dimensions and motivations, previously not widely adopted.

The current study is unique among the existing (published) research on gambling during the COVID-19 pandemic because it is one of the first to examine mental health changes as they relate to online gambling involvement at more than one point in time, while also considering financial impacts and motivation. Accordingly, this study provides insights into subgroups most vulnerable to mental health changes over time and variations in online gambling involvement. Those insights may be helpful when designing prevention approaches during times of reduced access to land-based gambling venues, such as during subsequent COVID-19 lockdowns. For example, our findings suggest that educational messaging during the pandemic (and similar events) may have the greatest utility in terms of reducing excessive gambling behavior if it focuses on some people's tendency to increase their gambling, especially if they are gambling to escape mental health problems (or the stress associated with the pandemic), or gambling as a means to make money (or make up for money lost as a result of the pandemic). Our findings also show that some people decreased their gambling during the pandemic. Understanding this group better may help with prevention efforts during the pandemic, but also be helpful post-pandemic. Indeed, there is currently a dearth of research on factors that lead to decreased gambling. Furthermore, results suggest that online gambling operators should (re)consider the information 
and resources provided to players during constricted gambling availability (Stark \& Robinson, 2021). For example, gambling operators should consider disseminating tailored information supporting players demonstrating play variation (determined via player account data).

This study addresses a need for repeated measurement research examining gambling impacts on public health phenomena (Price et al., 2021). Future research could try to replicate findings in international jurisdictions with different lockdown conditions and over longer time periods. Increased focus on identifying subgroups and addictive behaviors, integrating financial concerns and financially-focused self-concept, can improve our understanding of gambling problems and COVID-19 impacts.

\section{Limitations}

The current study only analyzed two time points separated by three months; though this may limit the breadth of our analyses, the data collected reflects a response to rapidly evolving changes in the pandemic. Recall bias may have affected results, and external validity may be limited as results are drawn from a single provincial jurisdiction. However, Ontario has a substantial foreign-born population ( 30\%; Ontario Ministry of Finance, 2017), which may mitigate social/cultural influences of more homogenous gambling populations. Some question items drafted for this survey may also have limited utility in assessing positive impacts associated with the pandemic, such as the Likert-scale question asking whether "my household income has been negatively affected by COVID19" (strongly disagree-strongly agree). Finally, the wording used to describe our analytic strategy varied slightly from that outlined in our pre-registration. For instance, instead of framing a hypothesis as a test of factors that may lead to "greater gambling involvement," we changed the wording to be one that tests factors that may lead to "variance in online gambling." We made this change because (after pre-registration) we realized wording the hypothesis as a test of "greater gambling involvement" neglects the fact that some people's gambling may decrease.

\section{Conclusion}

This study identified an online gambling cohort (subgroup 2) who varied the time, money, and games played during the COVID-19 pandemic. This subgroup's behavioral profile was associated with increased mental health problems over time, negative COVID-19 impacts on household income, stronger financially focused self-concepts, and elevated gambling problems. There is a critical need for further research on the intersection of online gambling, mental health, and financial security and motivation in other international settings. Such studies will help build consensus understanding on the pandemic effects-and more broadly stress and gambling access—related to comorbid health risks and socioeconomic determinants of health. To this end, a third wave of data has been collected and will be featured in future analyses of gambling and health trends.

Acknowledgements The authors acknowledge the technical support of Silas Xuereb and Mackenzie Dowson related to survey administration.

Funding This study was funded through a COVID-19 Rapid Response Research Grant (\#RSGRANT185594) awarded by Carleton University. 
All authors report no financial relationships with commercial interests or other conflicts of interest tied to this research.

AP has conducted independent research, evaluation, and reviews funded by the Carleton University (Canada), the Ontario Ministry of Health and Long-Term Care (Canada), the International Center for Responsible Gaming (USA), the Gambling Research Exchange Ontario (Canada), the Victorian Responsible Gambling Foundation (Australia), the Canadian Consortium for Gambling Research (Canada), the Canadian Gaming Association (Canada), the Ontario Lottery and Gaming Corporation, and other gambling operators in Canada, the UK, Italy, Australia, and Japan. AP has also received speaker/travel honoraria from the Canadian Center on Substance Use and Addiction (Canada), the Michigan Department of Health and Human Services (USA), and the New York Council on Problem Gambling (USA).

NT has received consulting fees from the gambling industry in Canada, New Zealand, the US, and the UK via GamRes Limited - a research and consultancy service that designs, implements, and evaluates responsible gambling strategies. NT has also received research contracts from Gambling Research Exchange (Canada). In addition, NT has received research funding as Principal Investigator or Co-Investigator from Gambling Exchange Ontario (Canada) and International Center for Responsible Gambling (USA).

MW has received research funding from Alberta Gambling Research Institute (Canada), British Columbia Lottery Corporation (Canada), Carleton University (Canada), Gambling Research Exchange Ontario (Canada), Manitoba Gambling Research Program (Canada), International Center for Responsible Gaming (USA), Ontario Lottery and Gaming (Canada), and Ontario Ministry of Health and Long-Term Care (Canada). MW has received speaker/travel honorarium from Alberta Liquor Gaming Commission (Canada), National Association for Gambling Studies (Australia), International Center for Responsible Gaming (USA), and Massachusetts Council on Compulsive Gambling (USA). He has received fees for academic services from Atlantic Lottery and Gaming Corporation (Canada), Gambling Research Exchange (Canada), National Center for Responsible Gaming (USA), New South Wales Government (Australia), Nova Scotia Gaming Corporation (Canada), Manitoba Gambling Research Program (Canada), Massachusetts Gambling Commission (USA), and Ontario Lottery and Gaming (Canada). MW has also received consulting fees from Alberta Liquor Gaming Commission (Canada), Atlantic Lottery and Gaming Corporation (Canada), British Columbia Lottery Corporation (Canada), GamRes (Canada), Massachusetts Gaming Commission (USA), Nova Scotia Gaming Corporation (Canada), and Ontario Lottery and Gaming (Canada).

IB has research funding from the Gambling Research Exchange Ontario (Canada) and the International Center for Responsible Gaming (USA). IB has received speaker/travel honorarium and fees for academic services from the Gambling Research Exchange Ontario (Canada) and the International Center for Responsible Gaming (USA).

SS has previously worked on prevention, research, or consultation projects funded by the Alcohol and Gaming Commission of Ontario (Canada), the Ontario Ministry of Health and Long-Term Care (Canada), Gambling Research Exchange Ontario (Canada/UK), the International Centre for Gaming Regulation (University of Las Vegas, USA), the International Center for Responsible Gaming (USA), Carleton University (Canada), the Camelot Group (UK), and other operators in Canada, the USA, and the UK.

\section{References}

Blaszczynski, A. (2009). Problem gambling: We should measure harm rather than 'cases.' Addiction, 104, 1070-1074. https://doi.org/10.1192/pb.34.6.259.

Brodeur, M., Audette-Chapdelaine, S., Savard, A. C., \& Kairouz, S. (2021). Gambling and the COVID19 pandemic: A scoping review. Progress in Neuro-Psychopharmacology and Biological Psychiatry, 111(April), 110389. https://doi.org/10.1016/j.pnpbp.2021.110389.

Browne, M., \& Rockloff, M. J. (2018). Prevalence of gambling-related harm provides evidence for the prevention paradox. Journal of Behavioral Addictions, 7(2), 410-422.

Browne, M., Langham, E., Rawat, V., Greer, N., Li, E., Rose, J., \& Rockloff, M., et al. (2016). “Assessing gambling-related harm in Victoria: A public health perspective." Melbourne, Australia. https://respo nsiblegambling.vic.gov.au/documents/69/Research-report-assessing-gambling-related-harm-in-vic.pdf.

Calado, F., \& Griffiths, M. D. (2016). Problem gambling worldwide: An update and systematic review of empirical research (2000-2015). Journal of Behavioral Addictions, 5(4), 592-613. https://doi.org/10. $1556 / 2006.5 .2016 .073$.

Dechant, K. (2014). Show me the money: Incorporating financial motives into the gambling motives questionnaire. Journal of Gambling Studies, 30, 949-965. https://doi.org/10.1007/s10899-013-9386-5.

Ferris, J., Wynne, H. (2001). The Canadian problem gambling index: Final report. http://www.greo.ca/ Modules/EvidenceCentre/files/Ferrisetal(2001)The_Canadian_Problem_Gambling_Index.pdf. 
Fluharty, M., Paul, E., \& Fancourt, D. (2020). "Predictors and patterns of gambling behaviour across the COVID-19 lockdown: Findings from a UK cohort study.” PsyArXiv Preprints December: 1-19. https:// doi.org/10.31234/osf.io/8qthw.

Frisone, F., Alibrandi, A., \& Settineri, S. (2020). Problem gambling during COVID-19. Mediterranean Journal of Clinical Psychology, 8(3), 1-15. https://doi.org/10.6092/2282-1619/mjcp-2457.

Gainsbury, S. M., Swanton, T. B., \& Blaszczynski, A. (2020). Impacts of the COVID-19 shutdown on gambling patterns in Australia. Journal of Addiction Medicine. https://doi.org/10.1097/ADM.0000000000 000793.

Gambling Commission. (2020). "Risks arising from COVID-19 and our response." News, Action and Statistics. 2020. https://www.gamblingcommission.gov.uk/news-action-and-statistics/Statistics-and-resea rch/Covid-19-research/Risks-arising-from-Covid-19-and-our-response.aspx.

Håkansson, A. (2020). Changes in gambling behavior during the COVID-19 pandemic: A web survey study in Sweden. International Journal of Environmental Research and Public Health, 17(4013), 1-16. https://doi.org/10.3390/ijerph17114013.

Hakansson, A., Fernandez-Aranda, F., Menchon, J. M., Potenza, M. N., \& Jimenez-Murcia, S. (2020). Gambling during the COVID-19 crisis: A cause for concern? Journal of Addiction Medicine. https://doi. org/10.1097/ADM.0000000000000690.

Hodgins, D. C., \& Stevens, R. M. G. (2021). The impact of COVID-19 on gambling and gambling disorder: Emerging data. Current Opinion in Psychiatry, 34(4), 332-343. https://doi.org/10.1097/YCO.00000 00000000709 .

Homans, W. (2012). The validity of the PHQ-9 and the GAD-7 for screening depressive and anxiety disorders in sick-listed workers. Retrieved January 18, 2022, https://dspace.library.uu.nl/bitstream/handle/ 1874/252945/Homans\%200472956.pdf?sequence=1\&isAllowed=y.

Kroenke, K., Spitzer, R. L., \& Williams, J. B. (2001). The PHQ-9: validity of a brief depression severity measure. Journal of General Internal Medicine, 16(9), 606-613. https://doi.org/10.1046/j.1525-1497. 2001.016009606.x.

Langham, E., Thorne, H., Browne, M., Donaldson, P., Rose, J., \& Rockloff, M. (2016). Understanding gambling related harm: A proposed definition, conceptual framework, and taxonomy of harms. BMC Public Health, 16(1), 80. https://doi.org/10.1186/s12889-016-2747-0.

Lewinsohn, P. M., \& Libet, J. (1972). Pleasant events, activity schedules, and depressions. Journal of Abnormal Psychology, 79(3), 291-95. https://doi.org/10.1037/h0033207.

Mallet, Jasmina, Dubertret, Caroline, \& Le, Yann. (2021). Addictions in the COVID-19 era: Current evidence, future perspectives a comprehensive review. Progress in Neuropsychopharmacology \& Biological Psychiatry, 106(March). https://doi.org/10.1016/j.pnpbp.2020.110070.

Neill, E., Meyer, D., Toh, W. L., Elizabeth, T., van Rheenen, A., Phillipou, E. J., \& Tan, and Susan Lee Rossell. (2020). Alcohol use in Australia during the early days of the COVID-19 pandemic: Initial results from the COLLATE project. Psychiatry and Clinical Neurosciences, 74(10), 542-549. https://doi.org/ 10.1111/pcn.13099.

Nicola, M., Alsafi, Z., Sohrabi, C., Kerwan, A., Al-Jabir, A., Iosifidis, C., Agha, M., \& Agha, R. (2020). The socio-economic implications of the coronavirus pandemic (COVID-19): A review. International Journal of Surgery, 78(January), 185-193. https://doi.org/10.1016/j.ijsu.2020.04.018.

Ontario Ministry of Finance. (2017). "2016 CENSUS HIGHLIGHTS: Factsheet 8.” 2017. https://www.fin. gov.on.ca/en/economy/demographics/census/cenhi16-8.html\#: :text=Ontario\%3A Highest Proportion of Foreign,the highest proportion among provinces.

Price, A. (2020). Online gambling in the midst of COVID-19: A nexus of mental health concerns, substance use and financial stress. International Journal of Mental Health and Addiction. https://doi.org/ 10.1007/s11469-020-00366-1.

Price, A., Hilbrecht, M., \& Billi, R. (2021). Charting a path towards a public health approach for gambling harm prevention. Journal of Public Health. https://doi.org/10.1007/s10389-020-01437-2.

Schellenberg, B., \& Mcgrath, D. S. (2016). The gambling motives questionnaire financial: Factor structure, measurement invariance, and relationships with gambling behaviour. International Gambling Studies, 16, 1-16. https://doi.org/10.1080/14459795.2015.1088559.

Sharman, Steve, Roberts, Amanda, \& Bowden-jones, Henrietta. (2021). Gambling and COVID-19: Initial findings from a UK sample. International Journal of Mental Health and Addiction. https://doi.org/10. 1007/s11469-021-00545-8.

Spitzer, R. L., Kroenke, K., Williams, J. J. B. W., \& Lo, B. (2006). A brief measure for assessing generalized anxiety disorder: the GAD-7. Archives of Internal Medicine, 166(10), 1092-1099. https://doi.org/ 10.1001/archinte.166.10.1092.

Stark, Sasha, and Janine Robinson. 2021. "Online gambling in unprecedented times: Risks and safer gambling strategies during the COVID-19 pandemic." Journal of Gambling Issues In Press. 
Sun, Y., Li, Y., Bao, Y., Meng, S., Sun, Y., Schumann, G., Kosten, T., Strang, J., Lin, Lu., \& Shi, J. (2020). Increased addictive internet and substance use behavior during the COVID-19 pandemic in China. American Journal on Addictions, 29, 268-270. https://doi.org/10.1111/ajad.13066.

Tabri, N., Will Shead, N., \& Wohl, M. J. A. (2017a). Me, myself, and money II: Relative deprivation predicts disordered gambling severity via delay discounting, especially among gamblers who have a financially focused self-concept. Journal of Gambling Studies. https://doi.org/10.1007/s10899-017-9673-7.

Tabri, N., Wohl, M. J. A., Eddy, K. T., \& Thomas, J. J. (2017b). Me, myself and money: Having a financially focused self-concept and its consequences for disordered gambling. International Gambling Studies, 17(1), 1-21. https://doi.org/10.1080/14459795.2016.1252414.

Tabri, N., Philander, K. S., Wood, R. T., \& Wohl, M. J. A. (2021a). Temporal measurement invariance of the financially focused self-concept construct. Journal of Gambling Issues, 48(48), 219-224. https://doi. org/10.4309/jgi.2021.48.11.

Tabri, Nassim, Xuereb, Silas, Cringle, Natalie, \& Clark, Luke. (2021b). Associations between financial gambling motives, gambling frequency and level of problem gambling: a meta-analytic review. Addiction. https://doi.org/10.1111/add.15642.

Tubadji, A., Webber, D. J., \& Boy, F. (2021). Cultural and economic discrimination by the Great Leveller. Regional Science Policy and Practice, 13(July), 198-216. https://doi.org/10.1111/rsp3.12456.

World Health Organization. (2021). WHO coronavirus disease (COVID-19) dashboard. https://covid19. who.int.

Publisher's Note Springer Nature remains neutral with regard to jurisdictional claims in published maps and institutional affiliations.

\section{Authors and Affiliations}

\section{Alex Price $^{1,2}$ (D) Nassim Tabri ${ }^{2,3} \cdot$ Sasha Stark ${ }^{1,2} \cdot$ Iris M. Balodis ${ }^{2,4,5}$. Michael J. A. Wohl' ${ }^{2,3}$}

1 Centre for the Advancement of Best Practices, The Responsible Gambling Council, 411 Richmond Street East Unit 205, Toronto, ON, Canada

2 Ontario Gambling Research Society, Toronto, ON, Canada

3 Department of Psychology, Carleton University, Ottawa, ON, Canada

4 Department of Psychiatry and Behavioural Neurosciences, McMaster University, Hamilton, ON, Canada

5 Peter Boris Centre for Addictions Research, McMaster University, Hamilton, ON, Canada 